\title{
Non-invasive ventilation
}

\author{
David Spence
}

\begin{abstract}
Summary
Nasal intermittent positive pressure ventilation is an effective treatment for nocturnal hypoventilation secondary to chest wall deformity or respiratory muscle weakness. Physicians should be aware that, in these groups of patients, disabling breathlessness can be alleviated and established cor pulmonale reversed by the technique.
\end{abstract}

Keywords: ventilation, nasal intermittent positive pressure ventilation

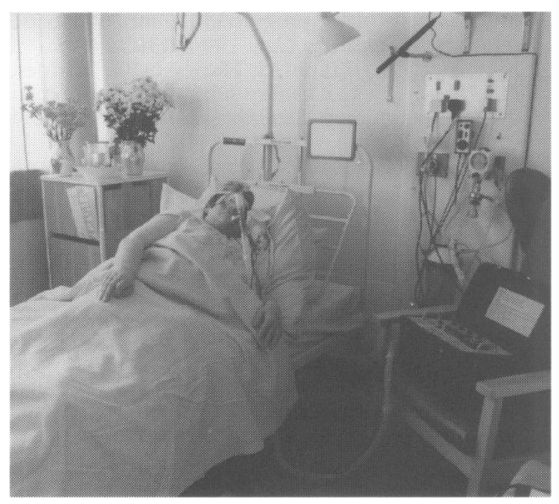

Figure 1 Patient with kyphoscoliosis receiving NIPPV

\begin{tabular}{|l|}
\hline Improvement in oxygenation \\
may be due to: \\
\hline - restoration of normal chemoreceptor \\
function \\
- respiratory muscle rest \\
- improved chest wall compliance \\
\hline
\end{tabular}

Box 1

Friarage Hospital, Northallerton, North Yorkshire DL6 1JG, UK D Spence
The advent of non-invasive ventilation by means of a nasal or face mask has? revolutionised the treatment of severe respiratory failure secondary to musclet weakness or chest wall deformity. Prior to nasal intermittent positive pressure ventilation (NIPPV) (figure 1), the treatment of these people was by externa: negative pressure ventilation using a tank ventilator (iron lung) (figure 2), a custom-built cuirass (figure 3), or a 'poncho' type ventilator; for patients withw respiratory muscle weakness the rocking bed was sometimes used. Alter $\overrightarrow{0}$ natively, positive pressure ventilation could be delivered by an endotracheaitube with all its attendant morbidity, both physical and psychological. NIPPVW can achieve better control of hypoventilation, is less cumbersome, and iso preferred by the patient, for these reasons the technique is increasing in popularity.

Currently, NIPPV has much greater acceptance in continental Europe where large numbers of patients are managed using assisted ventilation. In the UK its use is at present restricted to relatively few specialist centres.

\section{Theoretical considerations}

Breathing patterns are altered in response to changes in the level of consciousness. During sleep, muscle tone is reduced, particularly during rapic eye movement (REM) sleep when virtual atonia of the intercostal muscles occurs and breathing becomes almost exclusively diaphragmatic. In patients in whom the respiratory muscles are weak or who suffer a chest wall deformity this hypotonia inevitably results in nocturnal alveolar hypoventilation with resultante hypoxaemia and hypercapnia. Hypoxia is evident at first only during REM sleep but as muscle weakness increases or chest wall compliance decreaseso hypoventilation is present during progressively longer periods of sleep Ultimately, daytime hypoxaemia develops and pulmonary hypertension and cor pulmonale results.

In a minority of patients the nocturnal hypoxaemia can be controlled by the provision of supplemental oxygen. However, this does nothing tog prevent the underlying problem and has little effect on the unpleasant symptoms associated with alveolar hypoventilation. This is the rationale for assisted ventilation. Clinical studies have shown that nocturnal ventilation can improve oxygenation both by day and by night as well as alleviate the unpleasant symptoms associated with chest wall restriction or respiratoryo muscle weakness such as dyspnoea, reduced exercise tolerance, morning headache and poor sleep quality. There are three possible explanations for the improvement in daytime blood gases which results from nocturnak assisted ventilation (box 1). These are by restoring of normal chemo-o receptor function, by providing respiratory muscle rest, or by acting as physiotherapy on the chest wall and therefore improving chest walle compliance. The relative contributions of each of these mechanisms remains to be elucidated.

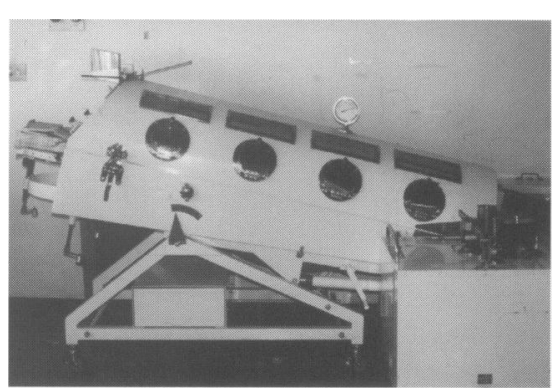

Figure 2 The tank ventilator or 'iron lung'

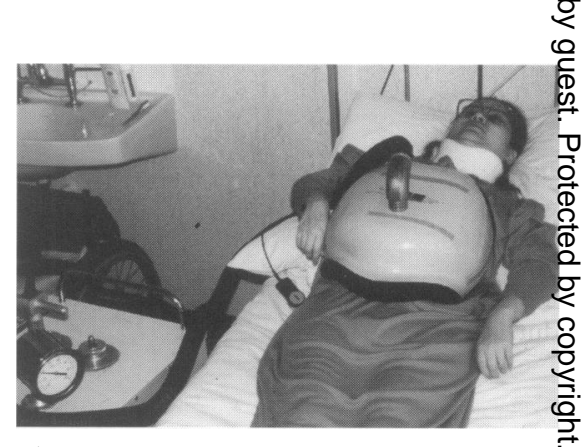

Figure 3 The Cuirass ventilator 


\begin{tabular}{|l|}
\hline Aims of NIPPV \\
\hline - improve sleep quality and ameliorate \\
morning headache \\
- reduce dyspnoea and improve \\
performance \\
- prevent cor pulmonale \\
\hline
\end{tabular}

Box 2

\begin{tabular}{|l|}
\hline Indications for non-invasive \\
ventilation \\
\hline - kyphoscoliosis: congenital or \\
paralytic \\
- sequellae of the surgical treatment of \\
tuberculosis: thoracoplasty, phrenic \\
crush \\
- ankylosing spondylitis (rare \\
complication) \\
- muscular weakness: muscular \\
dystrophies, myopathies \\
- neurological weakness: \\
poliomyelitis, motor neuron \\
disease, Guillain Barre syndrome; \\
bilateral phrenic nerve palsy \\
- disorders of ventilatory control: \\
congenital, associated with \\
neurological disease, obesity \\
\hline
\end{tabular}

\section{Box 3}

\section{The aims of NIPPV}

Assisted ventilation has three principal objectives (box 2). There are in the short term, to improve sleep quality and ameliorate morning headache, to reduce daytime dyspnoea and improve exercise performance and, in the longer term, to reverse or prevent the development of cor pulmonale.

\section{Which patients will benefit?}

NIPPV has been used in acute exacerbations of chronic obstructive pulmonary disease in order to avoid tracheal intubation or as an aid to weaning patients from mechanical ventilation. Selecting such patients for NIPPV is beyond the scope of this review, although recently published studies suggest its use will increase. It should be emphasised, however, that the use of NIPPV in such situations requires skilled nursing and physiotherapy staff and is not suitable for patients who are cerebrally obtunded.

Assisted ventilation may be used more chronically in a wide range of patients suffering from increased chest wall compliance or reduced respiratory muscle strength (box 3). Chest wall deformity, kyphoscoliosis both idiopathic and paralytic, together with thoracoplasty, form the majority of patients with chest wall deformity who develop respiratory failure. It is uncommon in ankylosing spondylitis, perhaps becuase the respiratory muscle geometry is well preserved in contrast to the other conditions. The age at which respiratory failure supervenes is variable and dependent on the degree of chest wall deformity together with the contribution of respiratory muscle weakness, however, it occurs most commonly in the forth or fifth decades of life.

Respiratory muscle weakness may be the presenting feature of muscular disease such as nemeline myopathy or acid maltase deficiency, although the involvement of the respiratory muscles is usually part of a more generalised process such as Duchenne muscular dystrophy. The decision to institute assisted ventilation in the presence of a generalised and often progressive disorder is sometimes difficult and should be undertaken only after full discussion with the patient and relatives. This discussion should cover the effectiveness of palliation of distressing symptoms such as breathlessness but should also include the possible increasing dependence upon the ventilator as the condition progresses. In practice the majority of patients derive such benefit from relief of breathlessness that they take to the technique readily.

Hypoventilation can occur as a result of disorders of central respiratory control mechanisms, either singly as in Ondine's Curse, or as part of more diffuse central nervous system damage. These patients form the ideal group for assisted ventilation as the underlying lung is normal and chest wall impedance is not increased. Central sleep apnoea also occurs in combination with obstructive sleep apnoea in patients with morbid obesity (Pickwickian syndrome). Obesity hypoventilation is commoner in women and attempts at sustained weight loss are often unsuccessful. Assisted ventilaion can overcome the hypoventilation and can be used in conjunction with nasal continuous positive airway pressure to control obstructive apnoeas. Unfortunately the mass loading of the chest wall frequently necessitates high inflation pressures which may be uncomfortable and poorly tolerated.

\section{When to institute NIPPV}

In patients with respiratory muscle weakness NIPPV is instituted when symptoms of carbon dioxide retention or postural breathlessness become troublesome. The time to institute treatment in thoracic cage deformity is more difficult. Most centres wait until significant nocturnal desaturation occurs (figure 4), but we sometimes implement treatment when there is a marked reduction in previously good exercise performance, since this usually precedes nocturnal desaturation.

\section{Establishing NIPPV}

NIPPV is best established electively, when undertaken as an emergency after the patient has already suffered a respiratory arrest and has been intubated and ventilated the outcome is much poorer, perhaps because sedative drugs make compliance a problem. In the elective setting, prior to the commencement of treatment, a full discussion of the technique with the patient should be undertaken. This should cover not only the likely benefits of the treatment but also the likely problems. This will encourage patients to persevere through the prolonged acclimatisation period while NIPPV is being established. 
1 Branthwaite MA. Non-invasive and domicillary ventilation: positive pressure techniques. Thorax 1991; 46: 208-12.

2 Cornette A, Mougel D. Ventilatory assistance via the nasal route; masks and fittings. Eur Respir Rev 1993; 3: 250-3.

3 Elliot MW. Non-invasive ventilation in chronic obstructive pulmonary disease. Lancet 1995; 333: $870-1$.

The patient is usually admitted to hospital where nursing and physiotherapy staff should be familiar with the treatment. The patient is shown the ventilator and nasal mask and is allowed to hold the mask over the nose himself when the ventilator is switched on. The initial inflation pressures are set low so as to minimise the initial discomfort. When comfortable with the mask the headstraps are secured and the patient is encouraged to adopt the most comfortable position for sleep. This is frequently sitting up or propped up lying on the side - positions which have been adopted over many years of respiratory difficulty during sleep. During the early part of establishing NIPPV it is usually? best to use the ventilator in the assist mode (the patient triggers all breaths) until: familar when a background ventilator rate may be set to prevent centralos apnoeas. The patient is encouraged to spend time during the day acclimatisingo to the technique, during this time the inflation pressures can be graduallyo increased. Overnight oximetry is performed to ensure that inflation pressures are adequate to control nocturnal hypoxaemia. In practice, approximately $15 \mathrm{~cm} \mathrm{H}_{2} \mathrm{O}$ is sufficient in patients with respiratory muscle weakness, whereas in chest wall deformity up to $30 \mathrm{~cm} \mathrm{H}_{2} \mathrm{O}$ may be required. In patients with established cor pulmonale, supplementary oxygen may be required. This can beadministered at a rate of $1-2 \mathrm{l} / \mathrm{min}$ into the nasal mask. As nocturnal hypoxaemia is controlled the pulmonary hypertension may regress ando supplementary oxygen can often be discontinued after one or two years.

Symptoms such as nasal dryness can be overcome by placing a humidifier in the circuit. Rhinorrhoea or nasal blockage are best treated by intranasalN ipratropium or topical corticosteroids.

4 Ellis ER, Bye PTP, Bruderer JW, Sullivan CE. Treatment of respiratory failure during sleep in patients with neuromuscular disease - positive pressure ventilation through a nose mask. $A m$ Rev Respir Dis 1987; 135: 148-52.

5 Heckmatt JZ, Loh L, Dubowitz V. Night time nasal ventilation in neuromuscular disease. Lancet 1990; 335: 579-82.
6 Hill NS, Eveloff SE, Carlisle CC, Goff SG Efficacy of nocturnal nasal ventilation in patients with restrictive thoracic disease. Am Rev Respir Dis 1992; 145: 365-71.

\section{The EuroTransMed Foundation Young Investigator Awards}

EuroTransMed is a Foundation of doctors providing live interactive educational programmes for doctors. It broadcasts by satellite up to 40 programmes a year to its network of over 200 European hospitals. The Foundation introduced its Young Investigator Awards in 1994 to encourage original research by doctors under 40 years of age. It is now pleased to announce the sixth round of the Awards, with a closing date of 18 October 1996, and submissions are invited in the research area of Diabetes.

Submissions should consist of new and original unpublished material, describing the work undertaken and the results obtained. Abstracts, not exceeding 200 words on A4 paper, should present information in the format of:

\section{- Background • Materials/methods • Results • Conclusion}

The abstracts will be reviewed by the Editorial Board of EuroTransMed, augmented by outside specialists where necessary, and successful candidates may be approached for further details. The winning authors will receive a cash award of ECU 1000 and, where appropriate, may be invited to present their results live in a EuroTransMed programme.

Abstracts must contain the names and addresses of all participants in the research, with the entrant's name underlined. Each abstract must contain a signed submission indicating that the material is new and has not been previously published (as of closing date), and must indicate the agreement of any coauthors. Abstracts failing to comply with these instructions will not be considered. All submissions will be acknowledged.

Candidates for the Young Investigator Award should submit two copies of their abstract to:

Dr HL Young, ChM FRCS

Chairman, Editorial Board EuroTransMed

c/o Department of Postgraduate Studies

University of Wales College of Medicine

Heath Park, Cardiff CF4 4XN, UK

Closing date for submissions: 18 October 1996

EuroTransMed programmes are approved for CME, subject to appropriate local organisation, by the Royal Colleges and the PGEA in the UK 